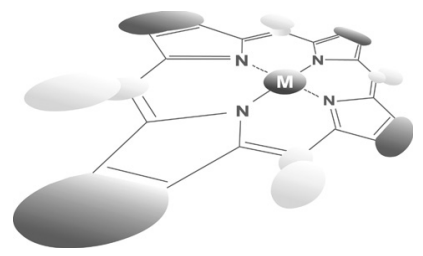

\title{
A novel potential nanophototherapeutic based on the assembly of an amphiphilic cationic $\beta$-cyclodextrin and an anionic porphyrin
}

\section{Antonino Mazzaglia*a, Norberto Micali ${ }^{\mathrm{b}}$, Valentina Villari ${ }^{\mathrm{b}}$, Roberto Zagamia, Rosa Maria Pennisi ${ }^{\mathrm{c}}$, Carmen Ortiz Mellet ${ }^{\mathrm{d}}$, José Manuel Garcia Fernández ${ }^{\mathrm{e}}$, Maria Teresa Sciortino ${ }^{c}$ and Luigi Monsù Scolaro ${ }^{a, c, f}$}

${ }^{a}$ CNR-ISMN Istituto per lo Studio dei Materiali Nanostrutturati, clo Dipartimento di Scienze Chimiche, Biologiche, Farmaceutiche ed Ambientali dell'Università di Messina, Viale Ferdinando Stagno d'Alcontres 31, 98166, Messina, Italy

${ }^{\mathrm{b}}$ CNR-IPCF Istituto per i Processi Chimico-Fisici, Viale Ferdinando Stagno d'Alcontres 37, 98158 Messina, Italy

${ }^{c}$ Dipartimento di Scienze Chimiche, Biologiche, Farmaceutiche ed Ambientali, Università di Messina, Viale Ferdinando Stagno d'Alcontres 98166, Messina, Italy

d Department of Organic Chemistry, Faculty of Chemistry, University of Sevilla, c/ Profesor García González 1, 41012 Sevilla, Spain

e Instituto de Investigaciones Químicas (IIQ), CSIC - Universidad de Sevilla, Avda. Américo Vespucio 49, E-41092 Sevilla, Spain

${ }^{\mathrm{f}}$ CIRCMSB, Unità di Messina, Italy

\section{Dedicated to Professor Claudio Ercolani on the occasion of his 80th birthday}

Received 10 January 2017

Accepted 24 March 2017

\begin{abstract}
The development of cyclodextrin nanoassemblies as useful carriers for photosensitizer drugs (PS) delivery in biological environment is a topic of increasing interest. In this paper, we present a spectroscopic investigation on a nanosystem based on an amphiphilic cationic $\beta$-cyclodextrin derivative (CD-N) and an anionic porphyrin (TPPS). Nanoassemblies were prepared by hydration of an organic film containing the two species. The system was characterized by complementary techniques such as UV-vis, stationary and time-resolved fluorescence, and Dynamic Light Scattering (DLS) at different TPPS/CD-N molar ratios. Time-resolved fluorescence data showed that, at all the investigated molar ratios, TPPS is present both as self-aggregated species and monomers forming supramolecular adducts with CD-N. Moreover, DLS measurements evidenced families of aggregates having hydrodynamic radii ranging between 50 and $350 \mathrm{~nm}$ and the size distribution profile depending on the TPPS/CD-N molar ratio. At the highest $\mathrm{CD}-\mathrm{N}$ concentration, the hydrodynamic radii of the aggregates were nearly the same as those of neat CD-N in the absence of TPPS $(50 \mathrm{~nm})$. No aging phenomena were registered, pointing out the high stability of these nanoassemblies in aqueous solution for at least a month. Preliminary studies on the internalization in tumoral cells and subsequent irradiation for PDT application were carried out. The results support the feasibility of these nanoaggregates to promote PS internalization in HeLa cells, inducing cell death upon visible light irradiation.
\end{abstract}

KEYWORDS: photosensitizers, cyclodextrins, nanoassemblies, fluorescence spectroscopy, photodamage, cancer cells.

\section{INTRODUCTION}

*Correspondence to: Antonino Mazzaglia, email: antonino. mazzaglia@ismn.cnr.it, tel/fax: +39 090-397-4108
The design of assemblies based on nanocarriers and photosensitizer drugs (PS) for controlled delivery and 
targeting in tumor tissue is a topic of increasing interest in nanooncology [1-4]. Photodynamic Therapy (PDT) is a selective treatment, which can be applied to solid tumors by delivering PS in the tissue and irradiating by an optical fiber using light of appropriate wavelength. PDT mechanism implies that light excites PS molecule from the ground state $\left({ }^{1} \mathrm{PS}\right)$ to an excited level, leading to ${ }^{1} \mathrm{PS} *$. The latter can convert to its triplet state $\left({ }^{3} \mathrm{PS}\right)$ by intersystem crossing. High quantum efficiency for this transition is a prerequisite for an efficient PS. Hence, ${ }^{3} \mathrm{PS}$ can transfer energy to the neighboring molecules and when this energy matches that required by molecular oxygen $\left(\mathrm{O}_{2}\right)$, it can produce singlet oxygen $\left({ }^{1} \mathrm{O}_{2}\right)$, one of the key species inducing controlled cell death. Cyclodextrins (CDs) are among the most common carriers utilized for research goals in PDT [5]. CDs are cyclic oligosaccharides composed by D-glucopyranosyl units linked through $\alpha-(1-4)$ bonds [6]. CD offers, either as molecule or in self-assembled form, functional constructs to bind PS guests by non-covalent interactions $[7,8]$. We have recently reported the design of different supramolecular assemblies of amphiphilic CDs with high efficacy for application in PDT and antimicrobial Photodynamic Therapy (aPDT) [9-12]. The physicochemical properties of a conventional anticancer molecule or NO-donor and a PS, respectively, can be conveniently modulated by dual entrapment in nanoassemblies based on amphiphilic or polymeric
CD with effects in the bio-fate of both drugs [13-15]. Actually, the design of novel nanophototherapeutics for PDT generally complies with efficient uptake of PS within tissues, cell membranes and/or intracellular components with production and diffusion of singlet oxygen in the neighboring areas (within 10-20 nm) [16] to the sites of action $[15,17,18]$. Anionic PS are less investigated in PDT, not so much for its singlet oxygen production, which is generally significant [9], but for the difficulty to cross the biological membranes which are negatively charged [19]. In this direction, an important issue concerns the use of cationic nanocarriers with high internalization efficacy [20]. CD-N represents a wellstudied amphiphilic cationic CD possessing excellent properties for cell-uptake and plasmid delivery [21]. The structure of the CD-N molecular scaffold has been shown to affect the self-aggregation properties and the ability to form mixed CD-DNA nanoparticles (CDplexes) [2224]. In the present paper we report on nanoassemblies based on amphiphilic cationic cyclodextrin (CD-N) and tetrakis(4-sulfonatophenyl)porphyrin (TPPS) (Scheme 1). TPPS/CD-N supramolecular assemblies were investigated by complementary spectroscopic techniques, such as UV-vis, steady-state and time-resolved fluorescence and anisotropy, and Dynamic Light Scattering (DLS). Moreover, their intracellular delivery together with preliminary results on cell survival for PDT application are here reported.
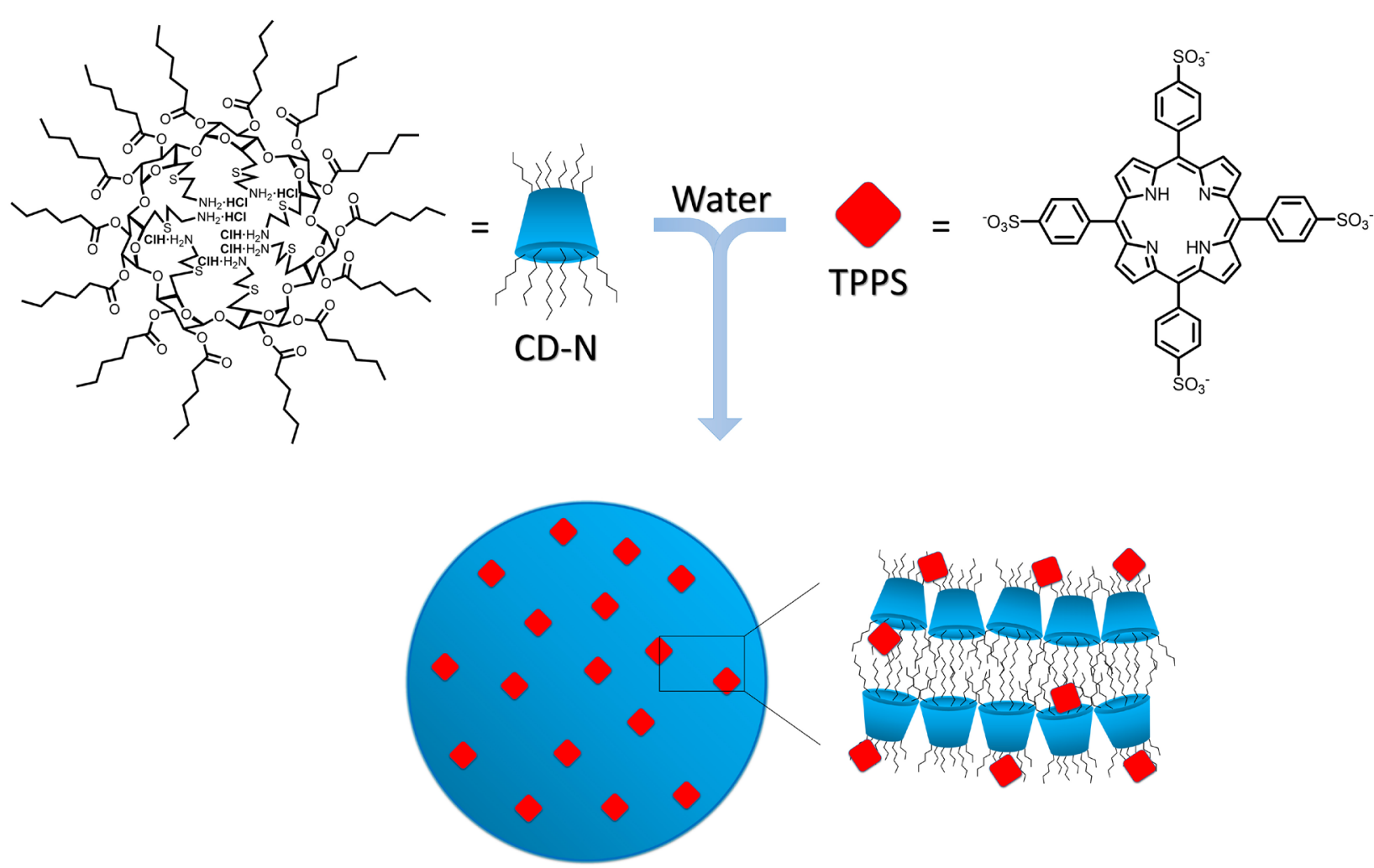

Scheme 1. Sketched view and molecular formulas of CD-N; TPPS and their CD-N/TPPS nanoassembly 


\section{EXPERIMENTAL}

\section{General}

All solvents were purified and dried using standard techniques. All other reagents were of the highest commercial grade available and were used as received or were purified via distillation or recrystallization when necessary. All solutions used for spectroscopic characterizations were prepared in pure microfiltered water (Galenica Senese, Siena, Italy) and analyzed at $298 \mathrm{~K}$. 5,10,15,20-Tetrakis (4-sulfonatophenyl)-21 H,23Hporphyrine (TPPS) was purchased from Sigma-Aldrich (Milan, Italy). Heptakis[6-(2-aminoethylthio)-6-deoxy-2,3di-O-hexanoyl]cyclomaltoheptaose $(\mathrm{CD}-\mathrm{N}, \mathrm{MW}=2900)$ was synthesized according to the reported procedure [21].

\section{Materials}

Nanoassemblies preparation. TPPS/CD-N nanoassemblies were prepared by hydration of an organic film of $\mathrm{CD}-\mathrm{N}$ with water at $\mathrm{pH} 6$ according to the conventional procedure used for similar nanosystems [25]. Briefly, a stock aqueous dispersion of CD-N $(400 \mu \mathrm{M})$ was prepared by hydration of an organic film, 20 min sonication in an ultrasound bath and equilibration at room temperature (rt). Aliquots of a stock aqueous solution of TPPS $(\cong 200 \mu \mathrm{M})$ were added to CD-N dispersion at different TPPS/CD-N molar ratios (1:1, 1:5 and 1:20, respectively) by increasing $\mathrm{CD}$ concentration, vortexing and equilibrating at rt. Each nanoassembly dispersion was slightly centrifuged to eliminate eventual not dispersed components. All the nanoassemblies dispersions for spectroscopic characterization were prepared by using $[\mathrm{TPPS}]=1.7 \mu \mathrm{M}$. An entrapment efficiency of $\cong 100 \%$ was estimated [25].

Methods. The UV-vis absorption spectra were collected on an Agilent model 8453 diode array spectrophotometer using $1 \mathrm{~cm}$ path length quartz cells. Steadystate fluorescence measurements were performed on a Jasco model FP-750 spectrofluorimeter. Emission spectra were collected using an excitation wavelength of $516 \mathrm{~nm}$ in a $1 \mathrm{~cm}$ path length quartz cell.

Time-resolved fluorescence. The light source for time-resolved fluorescence measurements is a polarized femtosecond Ti:Sa laser with duplication system, set at the wavelength of $500 \mathrm{~nm}$. Polarization analysis of the fluorescence signal collected at $650-660 \mathrm{~nm}$ is performed through an analyzer placed along the emission path. For the detection a monochromator $(1 / 8 \mathrm{~m})$ and a microchannelplate photomultiplier (100 ps rising-time) operating in single-photon counting regime are used. EG\&G electronic devices allow for pre-amplification, constant fraction discrimination, Time-to-Amplitude Conversion (TAC) and acquisition of fluorescence decay curves [26].

The collected data were analyzed using the nonlinear least-squares iterative reconvolution procedures, obtaining an instrumental resolution of about few tens of picoseconds.

Curve fitting was performed by using multiexponential decay law [27]:

$$
I(t)=I_{0} \sum_{i} A_{i} \exp \left(-t / \tau_{\mathrm{i}}\right)
$$

where $I(t)$ is total fluorescence decay curve, $I_{0}$ is the intensity at time zero, and $\mathrm{A}_{i}$ and $\tau_{i}$ are, respectively, the relative amplitude and lifetime of the $i$ th component, under the condition $\Sigma_{i} A_{i}=1$.

Fluorescence anisotropy decay is obtained from the difference between vertically, $I_{V V}$, and horizontally, $I_{V H}$, polarized emitted intensity and analyzed in terms of the following relation:

$$
D(t)=I_{V V}(t)-I_{V H}(t)=S(t)\left[r_{01} \exp \left(-t / \tau_{R}\right)+r_{\infty}\right]
$$

with $S(t)=I_{V V}+2 I_{V H}, \mathrm{r}_{01}$ the limiting anisotropy related to the rotational correlation time $\tau_{\mathrm{R}}$ and a plateau, $\mathrm{r}_{\infty}$, related to rotational correlation time longer than $20 \mathrm{~ns}$. Steadystate anisotropy is defined as $\mathrm{r}_{\text {staz }}=\left(I_{V V}-I_{V H}\right) /\left(I_{V V}+2 I_{V H}\right)$.

Dynamic light scattering. A $\mathrm{He}-\mathrm{Ne}$ laser beam (with power of $15 \mathrm{~mW}$ ), polarized orthogonal to the scattering plane, is focussed onto the sample. The scattered light is collected at $90^{\circ}$ by using two cooled $\left(-30^{\circ} \mathrm{C}\right)$ photomultipliers (in pseudocross correlation mode) and analyzed by a Malvern 4700 submicrometer particle analyzer which measures the scattered intensity autocorrelation function, $\mathrm{g}_{2}(t)$. For polydisperse dilute colloidal solutions it is possible to extract the average hydrodynamic radius of the scattering particles from the scattered intensity autocorrelation function through the following relation:

$$
\left[\mathrm{g}_{1}(t)\right]^{2}=\frac{\mathrm{g}_{2}(t)-1}{\alpha}=\exp (-2\langle\Gamma\rangle t)
$$

where $\alpha$ is a constant depending on the experimental setup and $\langle\Gamma\rangle=\frac{k_{B} T}{6 \pi \eta\left\langle R_{H}\right\rangle} Q^{2}$ is obtained by the cumulant analysis $[28,29]$ ( $\mathrm{Q}$ is the exchanged wavevector, $k_{\mathrm{B}}$ the Boltzmann's constant, $\mathrm{T}$ the absolute temperature and $\eta$ the solvent viscosity).

\section{Biological studies}

Cell culture. HeLa cells, originally obtained from the American Type Culture Collection (ATCC) were propagated at 1:6 ratio using Dulbecco's modification of Eagle's minimal essential medium (Lonza), supplemented with $10 \%$ fetal bovine serum (FBS).

Fluorescence microscopy analysis. HeLa cells were treated for $3 \mathrm{~h}$ with TPPS/CD-N nanoassemblies (at 1:5 molar ratio, TPPS $=1.7 \mu \mathrm{M}$ ) dispersed in culture medium, $\mathrm{pH}=7.4$ ). Hence cells were collected and layered on polylysinated slides. After $10 \mathrm{~min}$, the nuclei were labeled with HOECHST 33342 fluorescent DNA-binding 
dye at $5 \mu \mathrm{g} / \mathrm{mL}$ (Sigma). The intracellular fluorescence of TPPS/CD-N nanoassemblies was observed using a fluorescence microscope (Leitz, Wetzlar, Germany).

Photodynamic activity. HeLa cells were incubated with TPPS/CD-N nanoassemblies prepared at 1:5 molar ratio. Free TPPS and unloaded CD-N (prepared by using $[\mathrm{CD}-\mathrm{N}]=8.5 \mu \mathrm{M}$ ) were used as controls. Nanoassemblies and controls were dispersed in culture medium $(\mathrm{pH}=7.4)$. TPPS amount was fixed at $1.7 \mu \mathrm{M}$. After treatment, cells were collected, washed and re-suspended in PBS. Cells suspensions were then placed in a $1 \mathrm{~cm}$ spectrophotometric cuvette and irradiated by halogen lamp (Osram) for $30 \mathrm{~min}$. The irradiating beam was filter through an UV filter (Hoya glass type UV-34, cut-off: $340 \mathrm{~nm}$ ) in order to cut the UV component. A 1-cm cell filled with water was used to remove the IR-component. A light dose of $\cong 5$ joule $\times \mathrm{cm}^{-2}$ was evaluated. After irradiation, the percentage of dead cells was estimated by optical microscopy in a Burker chamber, using the trypan blue exclusion standard assay [30].

Statistical analysis. The results are reported as means \pm standard deviation and statistical calculations and graphical representation were done by One-way analysis of variance (ANOVA) using Prism software (GraphPad): $* P \leq 0.05, * * P<0.01, * * * P<0.001$.

\section{RESULTS AND DISCUSSION}

The interaction between porphyrin and cationic cyclodextrin was investigated in aqueous solutions by UV-vis and steady-state fluorescence. Figure 1 displays UV-vis spectra of TPPS/CD-N at different molar ratios. Free TPPS spectrum shows the typical B-band (Soret band) centered at $414 \mathrm{~nm}$, which becomes wider and more hypochromic at 1:1 TPPS/ CD-N molar ratio (trace b). By increasing the amount of carrier (to 1:5 PS/CD molar ratio), the B-band exhibits a bathochromic shift to $418 \mathrm{~nm}$ with a clear bump at lower wavelength (trace c).

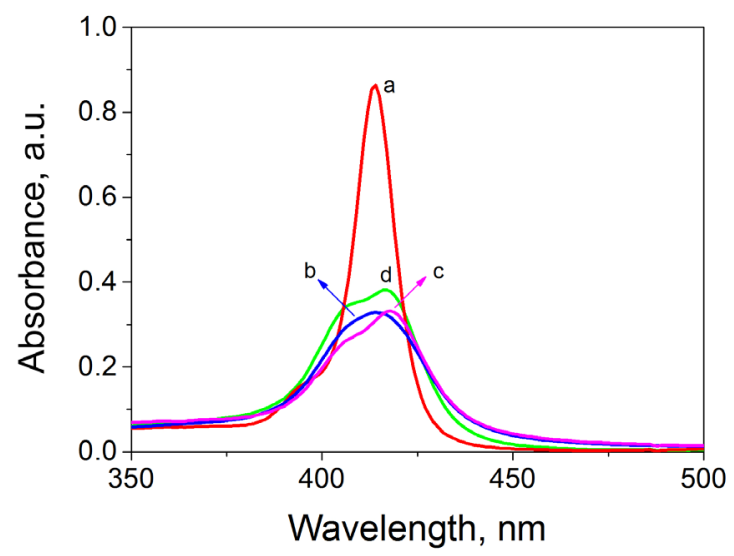

Fig. 1. UV-vis spectra of TPPS/CD-N nanoassemblies prepared at different molar ratio (TPPS $=1.7 \mu \mathrm{M}, \mathrm{T}=298 \mathrm{~K}, \mathrm{pH} \cong 6$ ). (a) Free TPPS, (b) 1:1, (c) 1:5, (d) 1:20 TPPS/CD-N molar ratios

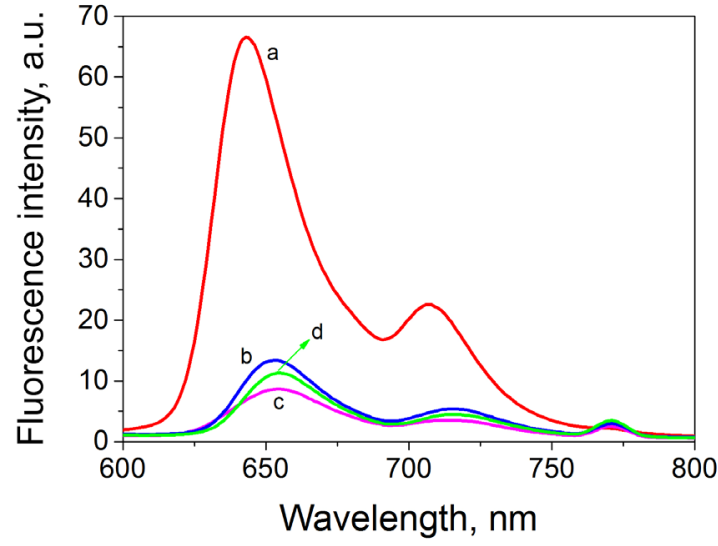

Fig. 2. Fluorescence emission spectra of TPPS/CD-N nanoassemblies $\left(\lambda_{\text {exc }}=516 \mathrm{~nm}\right)$ prepared at different molar ratio (TPPS $=1.7 \mu \mathrm{M}, \mathrm{T}=298 \mathrm{~K}, \mathrm{pH} \cong 6$ ). (a) Free TPPS, (b) 1:1, (c) 1:5, (d) 1:20 TPPS/CD-N molar ratios

At 1:20 molar ratio, the spectrum is more intense (than at higher molar ratio), exhibiting a band centered at $417 \mathrm{~nm}$ and maintaining the shoulder at $405 \mathrm{~nm}$ (trace d). The first band $(417 \mathrm{~nm})$ has been ascribed to TPPS/ CD-N supramolecular complexes, whereas the second one $(405 \mathrm{~nm})$ is presumably due to the presence of TPPS oligomers (i.e. $\mathrm{H}$ aggregates). These observations agree with a strong interaction between the porphyrin and the cyclodextrins network even at the higher molar ratios (1:1 and 1:5). However, the presence of the absorption band around $405 \mathrm{~nm}$ suggests the formation of oligomer species, which could affect the PDT efficacy.

Steady-state fluorescence emission spectra are shown in Fig. 2. Free TPPS typically exhibits a spectral profile composed by two bands, one centered at $643 \mathrm{~nm}$ and the other one at $708 \mathrm{~nm}$ (trace a). In TPPS/CD-N assemblies (at all the investigated molar ratios), these bands shifted to $\cong 652 \mathrm{~nm}$ and $716 \mathrm{~nm}$, respectively (traces b-d). Moreover, the fluorescence emission spectra of porphyrin/cyclodextrin nanoassemblies are remarkably less intense with respect to the free PS, presumably due to the interaction between the CD carrier and the porphyrin and to the formation of self-aggregated PS oligomers. Interestingly UV-vis and fluorescence emission spectra recorded on the TPPS/CD-N dispersions at lower molar ratios (1:5 and 1:20) after 30 days of storing at room temperature were practically unaltered (data not shown), suggesting an excellent stability of the supramolecular assemblies at the investigated concentrations.

Fluorescence lifetime measurements of TPPS in the absence of CD-N show a bi-exponential decay, ascribable to self-aggregated TPPS oligomers $\left(\tau_{1} \sim 1 \mathrm{~ns}\right)$ and to TPPS in monomeric form $\left(\tau_{2} \sim 10 \mathrm{~ns}\right)$ [31]. Accordingly, fluorescence anisotropy displays a fast rotational dynamics typical of the TPPS free monomer in solution (small rotational correlation time), and a much slower rotation (rotational correlation time longer than 
Table 1. Summary of the time-resolved fluorescence and DLS experiments ${ }^{\mathrm{a}}$

\begin{tabular}{lcccccccccc}
\hline TPPS/CD-N & $\tau_{1} \pm 0.1, \mathrm{~ns}$ & $\tau_{2} \pm 0.1, \mathrm{~ns}$ & $\mathrm{~A}_{1}, \%$ & $\mathrm{~A}_{2}, \%$ & $\tau_{\mathrm{R}} \pm 0.2, \mathrm{~ns}$ & $\mathrm{r}_{01}$ & $\mathrm{r}_{\infty}$ & $\mathrm{r}_{\text {staz }}$ & $\lambda_{\text {em }}, \mathrm{nm}$ & $\mathrm{R}_{\mathrm{H}}, \mathrm{nm} \pm 5 \%$ \\
\hline $1: 0$ & 1.2 & 10.0 & 50 & 50 & 0.3 & 0.035 & 0.025 & 0.03 & 650 & - \\
$1: 1$ & 1.1 & 7.1 & 70 & 30 & 1.3 & 0.02 & 0.02 & 0.03 & 655 & 340 \\
$1: 5$ & 1.2 & 6.2 & 70 & 30 & 1.9 & 0.01 & 0.03 & 0.04 & 660 & 150 \\
$1: 20$ & 1.3 & 5.5 & 65 & 35 & - & - & 0.04 & 0.04 & 660 & 50 \\
\hline
\end{tabular}

${ }^{a}$ All the parameters are defined in the Experimental section.

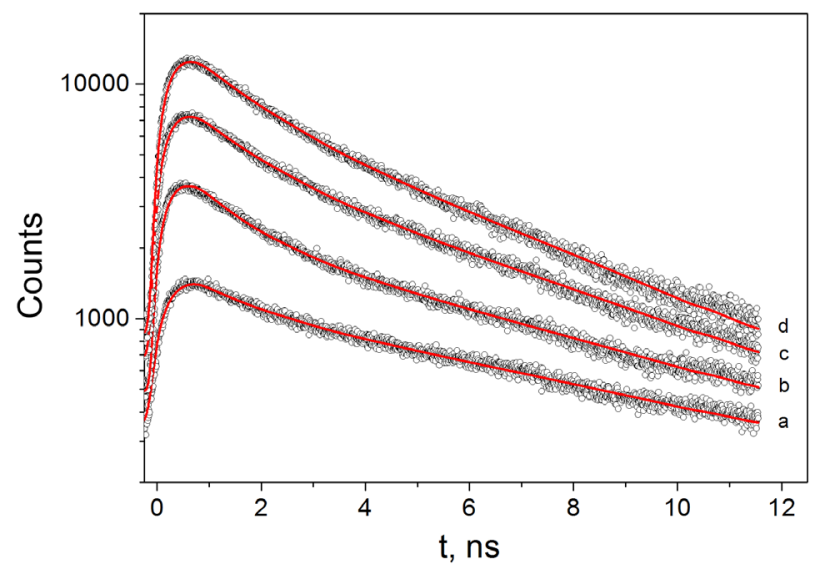

Fig. 3. Time-resolved fluorescence decays of: (a) free TPPS, (b) $1: 1$, (c) $1: 5$, (d) 1:20 TPPS/CD-N molar ratios ([TPPS] $=$ $1.7 \mu \mathrm{M}, \mathrm{T}=298 \mathrm{~K}, \mathrm{pH} \cong 6$ ). In order to make the graph more readable, each curve is scaled by a multiplicative factor $(1,1.5$, 3 , and 5 , respectively)

$20 \mathrm{~ns}$ ) consistent with the existence of aggregates. The shorter lifetime does not change upon addition of CD-N at any concentration, suggesting that the contribution from aggregates is still present; on the other hand, the longer lifetime becomes shorter and shorter as CD-N amount increases (see Fig. 3 and Table 1). This effect is due to the interaction between TPPS and CD-N. In addition, the rotational correlation time of the monomer slows down, furnishing a clear evidence of a static interaction due to the formation of supramolecular adducts.

It is worth to note that the hydrodynamic radius of TPPS/CD-N assemblies, observed by DLS, decreases towards the value of TPPS-unloaded CD-N nanoaggregates $(\sim 40 \mathrm{~nm})$ upon decreasing the TPPS/ CD-N molar ratio, as displayed in Fig. 4 and Table 1. This behaviour agrees with the dissolution of TPPS selfaggregates species in $\mathrm{CD} / \mathrm{PS}$ assemblies at high carrier concentration.

In order to investigate the potential of nanoassemblies based on CD-N/TPPS in PDT, preliminary cell uptake studies were carried out. HeLa cells were treated with nanoassemblies of CD-N/TTPS (at 1:5 molar ratio) for $3 \mathrm{~h}$ and analyzed by a double fluorescence microscopy method (see Fig. 5). Untreated HeLa cells were analyzed as control. The presence of TPPS within the cells was

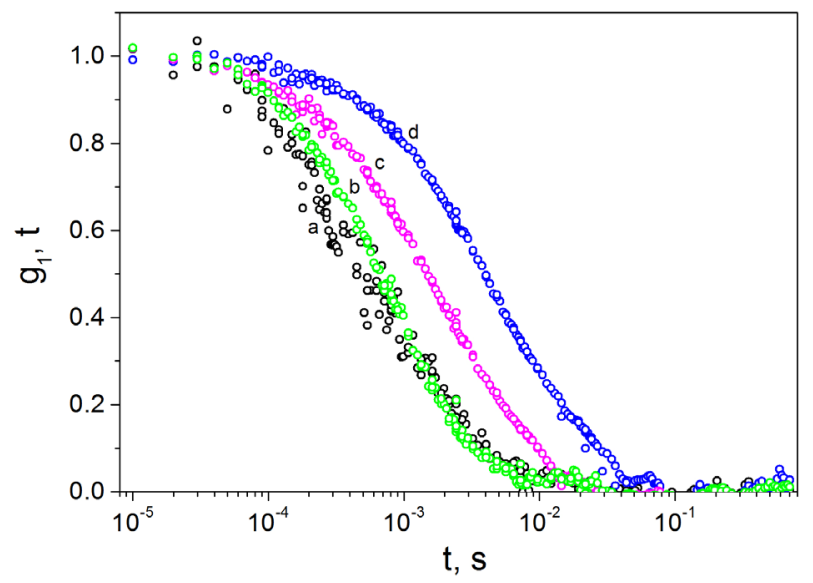

Fig. 4. DLS measurements (normalized scattered electric field autocorrelation functions measured at scattering angle of $90^{\circ}$ ) of CD-N (trace a) and TPPS/CD-N nanoassemblies prepared at different TPPS/CD-N molar ratio: (b) 1:20, (c) 1:5, (d) 1:1 TPPS/CD-N molar ratios $(\mathrm{TPPS}=1.7 \mu \mathrm{M}, \mathrm{T}=298 \mathrm{~K}, \mathrm{pH} \cong 6$ )

easily detected by the typical red fluorescence emission, while the location in the nuclear compartment was assessed by staining with the fluorescent HOECHST dye. Figure $5 d$ evidences a well detectable level of red fluorescence intensity within $\mathrm{HeLa}$ cells with respect to untreated cells (Fig. 5a), confirming the capability of cationic $\mathrm{CD}-\mathrm{N}$ to promote the intracellular delivery of TPPS. Figures $5 \mathrm{~b}$ and $5 \mathrm{e}$ show nuclear staining with HOECHST of untreated and treated cells, respectively, as revealed by the blue fluorescence emission of this dye. Figure $5 \mathrm{f}$ reports the merged image, i.e. the double fluorescence analysis derived from overlapping Fig. 5 ( $d$ and e). A detailed analysis of Fig. $5 \mathrm{f} v s$. the control in Fig. 5c, excludes the localization of porphyrins at the nuclear compartment level in HeLa cells.

After testing the feasibility of TPPS/CD-N to internalize in cancer cells, their photodynamic effect was preliminarily investigated. HeLa cells were treated for $3 \mathrm{~h}$ with free TPPS and TPPS/CD-N nanoassemblies at 1:5 molar ratio. The latter ratio was preferred than one with higher $\mathrm{CD}-\mathrm{N}$ concentration (i.e. 1:20) to minimize the dark toxicity. After treatment, samples were exposed to visible light for $30 \mathrm{~min}$ at rt. Figure 6 shows that cells treated with nanoassemblies at 1:5 molar ratio were 


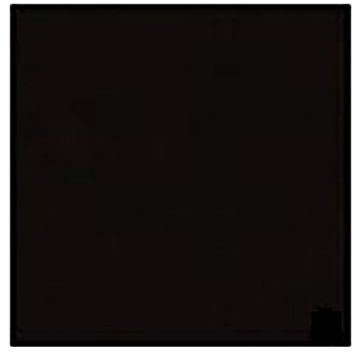

(a)

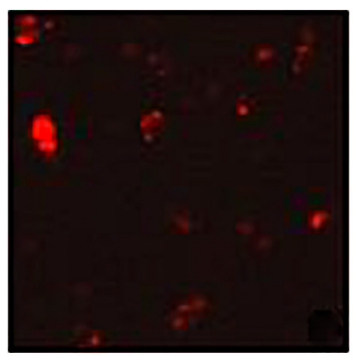

(d)

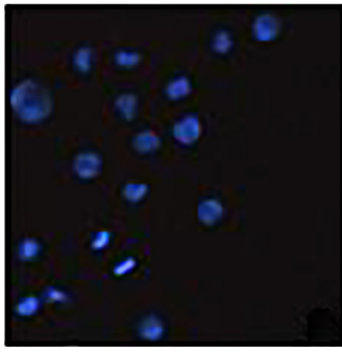

(b)

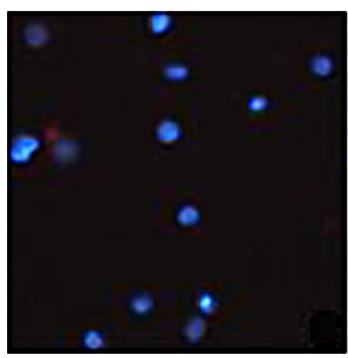

(e)

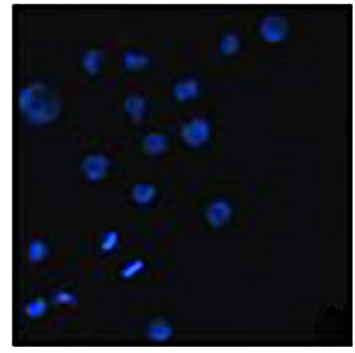

(c)

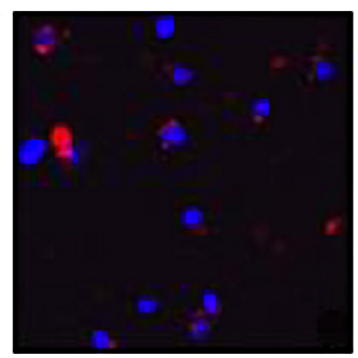

(f)

Fig. 5. Fluorescence microscopy analysis of HeLa cells treated for $3 \mathrm{~h}$ with TPPS/CD-N nanoassemblies (d-e) and untreated (a-c). HeLa cells were stained with HOECHST 33342 dye in phosphate buffer solution (10 mM, pH 7.4), and analyzed by fluorescence microscopy $(40 \mathrm{X})$ with a rhodamine filter ( $\mathrm{a}$ and $\mathrm{d})$, a DAPI filter ( $\mathrm{b}$ and e) or by merging images a and b, $\mathrm{d}$ and e, respectively (c and f)

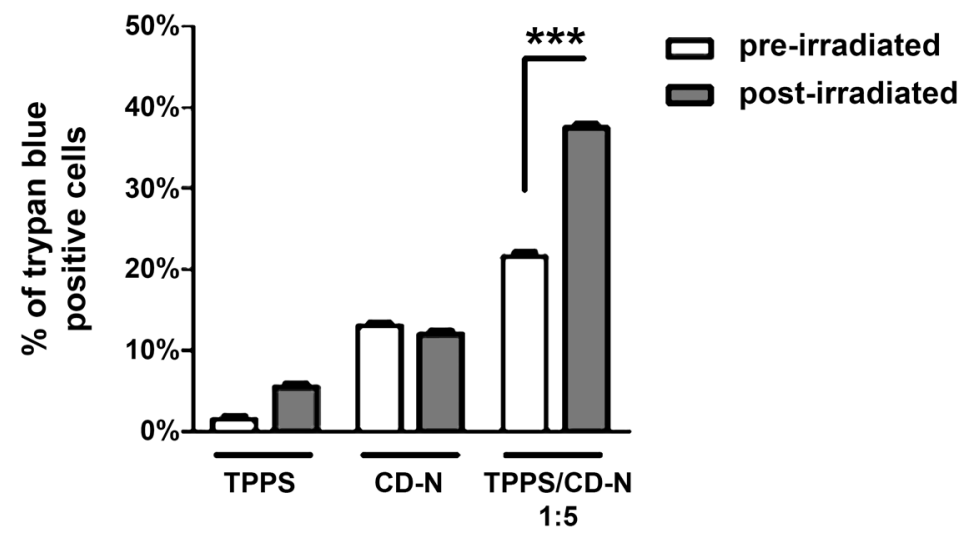

Fig. 6. Cell death percentage in HeLa using trypan blue assay. HeLa cells were treated with free TPPS (1.7 $\mu \mathrm{M})$, unloaded CD-N, as controls, and with TPPS/CD-N nanoassemblies prepared at 1:5 molar ratios, respectively. TPPS amount was fixed at $1.7 \mu \mathrm{M}$. Samples were exposed to Vis light irradiation for $30 \mathrm{~min}$ (see Experimental section for details)

responsive to irradiation-induced cell death $(\sim 40 \%$ of dead cells). However, it should be noticed that under our experimental conditions HeLa cells showed also a fair propensity to dark cell death caused by TPPS/CD-N in absence of irradiation ( $20 \%$ of dead cells). Interestingly, the cyclodextrin/PS nanosystem is more sensitive than free PS to produce photodamage, likely because of the higher porphyrin uptake due to the delivery action of CD carrier. Nonetheless an appreciable toxicity mediated by the CD carrier alone was found ( $\sim 10 \%$ of dead cells).

\section{CONCLUSION}

TPPS is non-covalently bounded in monomeric form (Soret band at $417 \mathrm{~nm}$ ) to the CD-N nanocarrier at TPPS/
CD-N molar ratio $\leq 1: 1$, although at all the investigated molar ratios a percentage of small oligomers of TPPS is present, as suggested by the shoulder at $405 \mathrm{~nm}$ in the UV-vis spectra, by quenching of the steady-stationary fluorescence emission spectra and by detection of shorter lifetimes in the time-resolved fluorescence decay, respectively. The formation of supramolecular assemblies between TPPS and CD-N nanocarrier is supported by time-resolved fluorescence anisotropy experiments, which shows a slowing down of rotational correlation time of monomer at increasing carrier loads. This behavior agrees with the dissolution of TPPS self-aggregates species in $\mathrm{CD}$ carrier forming CD/PS assemblies. Monomeric TPPS in the nanoassemblies might be responsible for the photodynamic damage 
since upon irradiation could produce higher ${ }^{1} \mathrm{O}_{2}$ yields as compared with the self-aggregated oligomers. The internalization of TPPS/CD-N (at 1:5 molar ratio) in HeLa cells and the subsequent effect upon irradiation for PDT application were preliminarily investigated; the data confirm the cell uptake of TPPS promoted by CD-N carrier and the photodamage upon irradiation, under our experimental conditions. The modulation of dark toxicity $v s$. the phodynamic effect of these nanoassemblies aimed to obtain an efficient and controlled delivery in tumoral tissues in vivo is the object of ongoing research in our laboratories. Furthermore, due to the excellent properties of $\mathrm{CD}-\mathrm{N}$ as plasmid carriers, a proper combination of photosensitiser and plasmid for dual delivery represents a future challenge.

\section{Acknowledgements}

The authors thank MIUR (PRIN 2010-2011 project no. 2010C4RM8) and CNR (Project ISMN-CNR: Materials and Dispositives for Health and Life Quality) for financial support. This study was also supported by the Spanish Ministerio de Economía y Competitividad (contract numbers SAF2016-76083-R and CTQ201564425-C2-1-R), the Junta de Andalucía (contract number FQM2012-1467 and the European Regional Development Funds (FEDER and FSE).

\section{REFERENCES}

1. Conte C, Ungaro F, Maglio G, Tirino P, Siracusano G, Sciortino MT, Leone N, Palma G, Barbieri A, Arra C, Mazzaglia A and Quaglia F. J. Controlled Release 2013; 167: 40-52.

2. Conte C, Ungaro F, Mazzaglia A and Quaglia F. Nano-Oncologicals: New Targeting and Delivery Approaches. Advances in Delivery Science and Technology, CRS Springer: New York, 2014.

3. Maiolino S, Moret F, Conte C, Fraix A, Tirino P, Ungaro F, Sortino S, Reddi E and Quaglia F. Nanoscale 2015; 7: 5643-5653.

4. Kemp JA, Shim MS, Heo CY and Kwon YJ. Adv. Drug Deliv. Rev. 2016; 98: 3-18.

5. Mazzaglia A, Micali N, Monsù Scolaro L, Sciortino MT, Sortino S and Villari V. J. Porphyrins Phthalocyanines 2010; 14: 661-677.

6. Crini G. Chem. Rev. 2014; 114: 10940-10975.

7. Kryjewski M, Goslinski T and Mielcarek J. Coord. Chem. Rev. 2015; 300: 101-120.

8. Mazzaglia A, Micali N and Monsù Scolaro L. In Molecular recognition by means of photophysical investigation; Cyclodextrins Materials: Photochemistry, Photophysics and Photobiology, Douhal A. (ed.) Elsevier: Amsterdam, 2006; pp. 203-222.

9. Sortino S, Mazzaglia A, Monsù Scolaro L, Marino Merlo F, Valveri V and Sciortino MT. Biomaterials 2006; 27: 4256-4265.
10. Mazzaglia A. Cyclodextrins in Pharmaceutics, Cosmetics, and Biomedicine: Current and Future Industrial Applications, Bilensoy E (ed.) John Wiley and Sons: New York, 2011; pp. 343-361.

11. Conte C, Scala A, Siracusano G, Leone N, Patane S, Ungaro F, Miro A, Sciortino MT, Quaglia F and Mazzaglia A. RSC Adv. 2014; 4: 43903-43911.

12. Ferro S, Jori G, Sortino S, Stancanelli R, Nikolov P, Tognon G, Ricchelli F and Mazzaglia A. Biomacromolecules 2009; 10: 2592-2600.

13. Kandoth N, Vittorino E, Sciortino MT, Parisi T, Colao I, Mazzaglia A and Sortino S. Chem.-Eur. J. 2012; 18: 1684-1690.

14. Conte C, Scala A, Siracusano G, Sortino G, Pennisi R, Piperno A, Miro A, Ungaro F, Sciortino MT, Quaglia F and Mazzaglia A. Colloids Surf., B 2016; 146: 590-597.

15. Fraix A, Kandoth N, Manet I, Cardile V, Graziano ACE, Gref R and Sortino S. Chem. Commun. 2013; 49: 4459-4461.

16. Kuimova MK, Yahioglu G and Ogilby PR. J. Am. Chem. Soc. 2009; 131: 332-340.

17. Voskuhl J, Kauscher U, Gruener M, Frisch H, Wibbeling B, Strassert CA and Ravoo BJ. Soft Matter. 2013; 9: 2453-2457.

18. Galstyan A, Kauscher U, Block D, Ravoo BJ and Strassert CA. ACS Appl. Mater. Interfaces 2016; 8: 12631-12637.

19. Zoltan T, Vargas F, López V, Chávez V, Rivas C and Ramírez ÁH. Spectrochim. Acta, Part A: Mol. Biomol. Spectrosc. 2015; 135: 747-756.

20. Jang W-D, Nakagishi Y, Nishiyama N, Kawauchi S, Morimoto Y, Kikuchi M and Kataoka K. J. Controlled Release 2006; 113: 73-79.

21. Díaz-Moscoso A, Balbuena P, Gómez-García M, Ortiz Mellet C, Benito JM, Le Gourriérec L, Di Giorgio C, Vierling P, Mazzaglia A, Micali N, Defaye J and García Fernández JM. Chem. Commun. 2008: 2001-2003.

22. Díaz-Moscoso A, Le Gourriérec L, Gómez-García M, Benito JM, Balbuena P, Ortega-Caballero F, Guilloteau N, Di Giorgio C, Vierling P, Defaye J, Ortiz Mellet C and García Fernández JM. Chem.Eur. J. 2009; 15: 12871-12888.

23. Ortiz Mellet C, García Fernández JM and Benito JM. Chem. Soc. Rev. 2011; 40: 1586-1608.

24. García Fernández JM, Benito JM and Ortiz Mellet C. Pure Appl. Chem. 2013; 85: 1825-1845.

25. Mazzaglia A, Bondì ML, Scala A, Zito F, Barbieri G, Crea F, Vianelli G, Mineo P, Fiore T, Pellerito C, Pellerito L and Costa MA. Biomacromolecules 2013; 14: 3820-3829.

26. Angelini N, Micali N, Villari V, Mineo P, Vitalini D and Scamporrino E. Phys. Rev. E 2005; 71: 021915.

27. Mazzaglia A, Valerio A, Villari V, Rencurosi A, Lay L, Spadaro S, Monsu Scolaro L and Micali N. New J. Chem. 2006; 30: 1662-1668. 
28. Villari V, Mazzaglia A, Darcy R, O’Driscoll CM and Micali N. Biomacromolecules 2013; 14: 811-817.

29. Faraone A, Magazù S, Maisano G, Ponterio R and Villari V. Macromolecules 1999; 32: 1128-1133.
30. Hoskins JM. Virological procedures, Butterworth \& Co Publishers Ltd: London, 1967.

31. Romeo A, Castriciano MA, Zagami R, Pollicino G, Monsu Scolaro L and Pasternack RF. Chem. Sci. 2017; 8: 961-967. 